\title{
The growth rate and dimension theory of beta-expansions
}

\author{
Simon Baker
}

November 21, 2018

\begin{abstract}
In a recent paper of Feng and Sidorov they show that for $\beta \in$ $\left(1, \frac{1+\sqrt{5}}{2}\right)$ the set of $\beta$-expansions grows exponentially for every $x \in$ $\left(0, \frac{1^{2}}{\beta-1}\right)$. In this paper we study this growth rate further. We also consider the set of $\beta$-expansions from a dimension theory perspective.
\end{abstract}

\section{Introduction}

Let $1<\beta<2$ and $I_{\beta}=\left[0, \frac{1}{\beta-1}\right]$. Each $x \in I_{\beta}$ can be expressed as

$$
x=\sum_{n=1}^{\infty} \frac{\epsilon_{n}}{\beta^{n}}
$$

for some $\left(\epsilon_{n}\right)_{n=1}^{\infty} \in\{0,1\}^{\mathbb{N}}$. We call such a sequence a $\beta$-expansion for $x$. We define

$$
\Sigma_{\beta}(x)=\left\{\left(\epsilon_{n}\right)_{n=1}^{\infty} \in\{0,1\}^{\mathbb{N}}: \sum_{n=1}^{\infty} \frac{\epsilon_{n}}{\beta^{n}}=x\right\} .
$$

In [2] it is shown that for $\beta \in\left(1, \frac{1+\sqrt{5}}{2}\right)$ and $x \in\left(0, \frac{1}{\beta-1}\right)$ the set $\Sigma_{\beta}(x)$ is uncountable. In [6, 7] the author considers the case where $\beta \in\left[\frac{1+\sqrt{5}}{2}, 2\right)$. They show that for Lebesgue almost every $x \in I_{\beta}$ the set $\Sigma_{\beta}(x)$ is uncountable. To describe the growth rate of $\beta$-expansions we consider the following. Let

$\mathcal{E}_{k}(x, \beta)=\left\{\left(\epsilon_{1}, \ldots, \epsilon_{k}\right) \in\{0,1\}^{k} \mid \exists\left(\epsilon_{k+1}, \epsilon_{k+2}, \ldots\right) \in\{0,1\}^{\mathbb{N}}: \sum_{n=1}^{\infty} \frac{\epsilon_{n}}{\beta^{n}}=x\right\}$,

we define an element of $\mathcal{E}_{k}(x, \beta)$ to be a $k$-prefix for $x$. Moreover, we let

$$
\mathcal{N}_{k}(x, \beta)=\# \mathcal{E}_{k}(x, \beta)
$$


and define the growth rate of $\mathcal{N}_{k}(x, \beta)$ to be

$$
\lim _{k \rightarrow \infty} \frac{\log _{2} \mathcal{N}_{k}(x, \beta)}{k}
$$

when this limit exists. When this limit doesn't exist we can consider the lower and upper growth rates of $\mathcal{N}_{k}(x, \beta)$, these are defined to be

$$
\liminf _{k \rightarrow \infty} \frac{\log _{2} \mathcal{N}_{k}(x, \beta)}{k} \text { and } \limsup _{k \rightarrow \infty} \frac{\log _{2} \mathcal{N}_{k}(x, \beta)}{k}
$$

respectively.

In this paper we also consider $\Sigma_{\beta}(x)$ from a dimension theory perspective. We endow $\{0,1\}^{\mathbb{N}}$ with the metric $d(\cdot, \cdot)$ defined as follows:

$$
d(x, y)= \begin{cases}2^{-n(x, y)} & \text { if } x \neq y, \text { where } n(x, y)=\inf \left\{i: x_{i} \neq y_{i}\right\} \\ 0 & \text { if } x=y .\end{cases}
$$

We consider the Hausdorff dimension of $\Sigma_{\beta}(x)$ with respect to this metric. It is a simple exercise to show the following inequalities hold:

$$
\operatorname{dim}_{H}\left(\Sigma_{\beta}(x)\right) \leq \liminf _{k \rightarrow \infty} \frac{\log _{2} \mathcal{N}_{k}(x, \beta)}{k} \leq \limsup _{k \rightarrow \infty} \frac{\log _{2} \mathcal{N}_{k}(x, \beta)}{k} .
$$

In [4] the following theorem was shown to hold.

Theorem 1.1. Let $\beta \in\left(1, \frac{1+\sqrt{5}}{2}\right)$. There exists $\kappa(\beta)>0$ such that

$$
\liminf _{k \rightarrow \infty} \frac{\log _{2} \mathcal{N}_{k}(x, \beta)}{k} \geq \kappa(\beta) \text { for all } x \in\left(0, \frac{1}{\beta-1}\right) .
$$

Here $\kappa(\beta)$ is given explicitly by the formula

$$
\kappa(\beta)= \begin{cases}\frac{1}{2}\left(\left[\log _{\beta}\left(\frac{\beta^{2}-1}{1+\beta-\beta^{2}}\right)\right]+1\right)^{-1} & \text { if } \beta>\sqrt{2} \\ \frac{1}{2}\left(\left[\log _{\beta}\left(\frac{1}{\beta-1}\right)\right]+1\right)^{-1} & \text { if } \beta \leq \sqrt{2} .\end{cases}
$$

The growth rate of $\mathcal{N}_{k}(x, \beta)$ is addressed from the measure theoretic point of view in [5]. The following result is implicit.

Theorem 1.2. For almost every $\beta \in(1,2)$ and for almost every $x \in I_{\beta}$,

$$
\limsup _{k \rightarrow \infty} \frac{\log _{2} \mathcal{N}_{k}(x, \beta)}{k}=\log _{2}\left(\frac{2}{\beta}\right) .
$$

Moreover, for almost every $\beta \in(1, \sqrt{2})$ and for almost every $x \in I_{\beta}$

$$
\lim _{k \rightarrow \infty} \frac{\log _{2} \mathcal{N}_{k}(x, \beta)}{k}=\log _{2}\left(\frac{2}{\beta}\right) .
$$


We remark that the bounds given in Theorem 1.1 are somewhat weak. We observe that $\kappa(\beta) \rightarrow 0$ as $\beta \rightarrow 1$, contrary to what we would expect. As $\beta \rightarrow 1$ we would expect the number of $k$-prefixes for $x$ to grow and the growth rate of $\mathcal{N}_{k}(x, \beta)$ to increase. In this paper we show that the following theorem holds.

Theorem 1.3. There exists a strictly decreasing sequence $\left(\omega_{m}\right)_{m=1}^{\infty}$ converging to 1 , such that, if $\beta \in\left(1, \omega_{m}\right]$ then

$$
\operatorname{dim}_{H}\left(\Sigma_{\beta}(x)\right) \geq \frac{2 m}{2 m+1} \text { for all } x \in\left(0, \frac{1}{\beta-1}\right) .
$$

As an immediate corollary of Theorem 1.3 we have that the infimum of $\operatorname{dim}_{H}\left(\Sigma_{\beta}(x)\right) \rightarrow 1$ as $\beta \rightarrow 1$, i.e.

$$
\inf _{x \in\left(0, \frac{1}{\beta-1}\right)}\left\{\operatorname{dim}_{H}\left(\Sigma_{\beta}(x)\right)\right\} \rightarrow 1
$$

as $\beta \rightarrow 1$. By (11) similar statements hold for the lower and upper growth rate of $\mathcal{N}_{k}(x, \beta)$.

We also improve on the bounds given in Theorem 1.1. We show that the following theorem holds.

Theorem 1.4. There exists a strictly increasing sequence $\left(\lambda_{m}\right)_{m=1}^{\infty}$ converging to $\frac{1+\sqrt{5}}{2}$, such that, for $\beta \in\left(1, \lambda_{m}\right]$

$$
\operatorname{dim}_{H}\left(\Sigma_{\beta}(x)\right) \geq \frac{1}{m+2} \text { for any } x \in\left(0, \frac{1}{\beta-1}\right) .
$$

In Section 2 we prove Theorem 1.3 and in Section 3 we prove Theorem 1.4, Theorem 1.4 will follow by a similar argument to Theorem 1.3. In Section 4 we give some bounds for the upper growth rate of $\mathcal{N}_{k}(x, \beta)$ and in Section 5 we use our results to obtain bounds for the local dimension of Bernoulli convolutions.

\section{Proof of Theorem 1.3}

To prove Theorem 1.3 we devise an algorithm for generating $\beta$-expansions. We then show that the Hausdorff dimension of the set of expansions generated by this algorithm is greater than or equal to $\frac{2 m}{2 m+1}$ for $\beta \in\left(1, \omega_{m}\right]$. Before giving details of this algorithm we provide a useful reinterpretation of $\mathcal{N}_{k}(x, \beta)$ and define the sequence $\left(\omega_{m}\right)_{m=1}^{\infty}$. 


\section{1 $\quad B_{k}(x, \beta)$ and the sequence $\left(\omega_{m}\right)_{m=1}^{\infty}$}

\subsubsection{Reinterpretation of $\mathcal{N}_{k}(x, \beta)$}

Fix $T_{0, \beta}(x)=\beta x$ and $T_{1, \beta}(x)=\beta x-1$. We let

$$
\Omega_{k}=\left\{a=\left(a_{n}\right)_{n=1}^{k} \in\left\{T_{0, \beta}, T_{1, \beta}\right\}^{k}\right\}
$$

Suppose $x \in I_{\beta}$ and $a \in \Omega_{k}$, we denote $a_{k} \circ a_{k-1} \circ \ldots \circ a_{1}(x)$ by $a(x)$. We let

$$
|a|_{0}=\#\left\{1 \leq n \leq k: a_{n}=T_{0, \beta}\right\}
$$

and

$$
|a|_{1}=\#\left\{1 \leq n \leq k: a_{n}=T_{1, \beta}\right\}
$$

Finally we define

$$
T_{k}(x, \beta)=\left\{a \in \Omega_{k}: a(x) \in I_{\beta}\right\}
$$

and

$$
B_{k}(x, \beta)=\# T_{k}(x, \beta) .
$$

Proposition 2.1. $\mathcal{N}_{k}(x, \beta)=B_{k}(x, \beta)$

Proof. Following [4] we observe that

$$
\begin{aligned}
\mathcal{E}_{k}(x, \beta) & =\left\{\left(\epsilon_{1}, \ldots, \epsilon_{k}\right) \in\{0,1\}^{k}: x-\frac{1}{\beta^{k}(\beta-1)} \leq \sum_{n=1}^{k} \frac{\epsilon_{n}}{\beta^{n}} \leq x\right\} \\
& =\left\{\left(\epsilon_{1}, \ldots, \epsilon_{k}\right) \in\{0,1\}^{k}: 0 \leq x-\sum_{n=1}^{k} \frac{\epsilon_{n}}{\beta^{n}} \leq \frac{1}{\beta^{k}(\beta-1)}\right\} \\
& =\left\{\left(\epsilon_{1}, \ldots, \epsilon_{k}\right) \in\{0,1\}^{k}: 0 \leq \beta^{k} x-\sum_{n=1}^{k} \epsilon_{n} \beta^{k-n} \leq \frac{1}{\beta-1}\right\} \\
& =\left\{\left(\epsilon_{1}, \ldots, \epsilon_{k}\right) \in\{0,1\}^{k}: 0 \leq\left(T_{\epsilon_{1}, \beta}, \ldots T_{\epsilon_{k}, \beta}\right)(x) \leq \frac{1}{\beta-1}\right\} .
\end{aligned}
$$

Our result follows immediately.

By Proposition 2.1 we can identify elements of $T_{k}(x, \beta)$ with elements of $\mathcal{E}_{k}(x, \beta)$, as such we also define an element of $T_{k}(x, \beta)$ to be a $k$-prefix of $x$. To help with our later calculations we include the following technical lemmas. 
Lemma 2.2. Let $k, n \in \mathbb{N}$, then

$$
T_{1, \beta}^{k}\left(\frac{\beta^{n}}{\beta^{2}-1}\right)=\frac{\beta^{n+k}-\beta^{k+1}-\beta^{k}+\beta+1}{\beta^{2}-1} .
$$

The proof of this lemma is trival and hence ommited.

Lemma 2.3. Assume $a \in \Omega_{2 k+1}$ and $|a|_{0} \geq k+1$. Then for all $x \in \mathbb{R}$

$$
a(x) \geq(\overbrace{T_{1, \beta}, \ldots, T_{1, \beta}}^{k} \overbrace{T_{0, \beta}, \ldots, T_{0, \beta}}^{k+1})(x) .
$$

Similarly suppose $|a|_{1} \geq k+1$ then

$$
a(x) \leq(\overbrace{T_{0, \beta}, \ldots, T_{0, \beta}}^{k} \overbrace{T_{1, \beta}, \ldots, T_{1, \beta}}^{k+1})(x) .
$$

Proof. Suppose $a \in \Omega_{2 k+1}$ and $|a|_{0} \geq k+1$. By a simple calculation we have

$$
a(x)=\beta^{2 k+1} x-\sum_{n=1}^{2 k+1} \chi_{n}(a) \beta^{2 k+1-n},
$$

where $\chi_{n}(a)=1$ if $a_{n}=T_{1, \beta}$ and 0 otherwise. Since $|a|_{0} \geq k+1$ we have that $\chi_{n}(a)=1$ for at most $k$ different values of $n$. It follows that

$$
a(x) \geq \beta^{2 k+1} x-\beta^{2 k}-\ldots-\beta^{k+1} .
$$

However,

$$
\beta^{2 k+1} x-\beta^{2 k}-\ldots-\beta^{k+1}=(\overbrace{T_{1, \beta}, \ldots, T_{1, \beta}}^{k}, \overbrace{T_{0, \beta}, \ldots, T_{0, \beta}}^{k+1})(x)
$$

and our results follows. The second inequality is proved similarly.

\subsubsection{The sequence $\left(\omega_{m}\right)_{m=1}^{\infty}$}

We now define our sequence $\left(\omega_{m}\right)_{m=1}^{\infty}$. To each $m \in \mathbb{N}$ we associate the polynomials:

$$
\begin{aligned}
& P_{m}^{1}(x)=x^{4 m+3}-x^{2 m+2}-x^{m+2}-x^{m+1}+x+1 \\
& P_{m}^{2}(x)=x^{2 m+3}-x^{2 m+2}-x^{2}+1 \\
& P_{m}^{3}(x)=x^{2 m+3}-x-1 .
\end{aligned}
$$

We define $\omega_{m}^{(i)}$ to be the smallest real root of $P_{m}^{i}(x)$ greater than 1 . Clearly $P_{m}^{3}(x)$ has a real root greater than 1 . To see that $P_{m}^{1}(x)$ and $P_{m}^{2}(x)$ both have a real root greater than 1 we observe that $P_{m}^{i}(1)=0$ and $\left(P_{m}^{i}\right)^{\prime}(1)<0$ for $i=1,2$. We define $\omega_{m}=\min \left\{\omega_{m}^{(1)}, \omega_{m}^{(2)}, \omega_{m}^{(3)}\right\}$. We now state some properties of $\left(\omega_{m}\right)_{m=1}^{\infty}$ that will be useful in our later analysis. 
Property 2.4. For $\beta \in\left(1, \omega_{m}\right]$

$$
\beta^{4 m+3}-\beta^{2 m+2}-\beta^{m+2}-\beta^{m+1}+\beta+1 \leq 0 .
$$

Proof. This follows since $\omega_{m} \leq \omega_{m}^{(1)}, P_{m}^{1}(1)=0$ and $\left(P_{m}^{1}\right)^{\prime}(1)<0$.

Property 2.5. For $\beta \in\left(1, \omega_{m}\right]$

$$
T_{0, \beta}^{2 m+1}\left(\frac{\beta}{\beta^{2}-1}\right)=\frac{\beta^{2 m+2}}{\beta^{2}-1}
$$

and $\frac{\beta^{2 m+2}}{\beta^{2}-1} \in I_{\beta}$.

Proof. It suffices to show that

$$
\frac{\beta^{2 m+2}}{\beta^{2}-1} \leq \frac{1}{\beta-1}
$$

This is equivalent to

$$
\beta^{2 m+3}-\beta^{2 m+2}-\beta^{2}+1 \leq 0 .
$$

This is true for $\beta \in\left(1, \omega_{m}\right]$ since $\omega_{m} \leq \omega_{m}^{(2)}, P_{m}^{2}(1)=0$ and $\left(P_{m}^{2}\right)^{\prime}(1)<0$.

Property 2.6. For $\beta \in\left(1, \omega_{m}\right]$

$$
T_{1, \beta}^{2 m+1}\left(\frac{\beta}{\beta^{2}-1}\right)=\frac{-\beta^{2 m+1}+\beta+1}{\beta^{2}-1},
$$

by Lemma 2.2. Moreover $\frac{-\beta^{2 m+1}+\beta+1}{\beta^{2}-1} \in I_{\beta}$.

Proof. It suffices to show that

$$
\frac{-\beta^{2 m+1}+\beta+1}{\beta^{2}-1} \geq 0 \text {. }
$$

This is equivalent to

$$
\beta^{2 m+1}-\beta-1 \leq 0 .
$$

This is true for $\beta \in\left(1, \omega_{m}\right]$ since $\omega_{m} \leq \omega_{m}^{(3)}$ and $P_{m}^{3}(1)<0$.

Property 2.7. The sequence $\left(\omega_{m}\right)_{m=1}^{\infty}$ is strictly decreasing and converging to 1. This follows from the fact that $\left(\omega_{m}^{(i)}\right)_{m=1}^{\infty}$ is strictly decreasing and converging to 1 for $i=1,2,3$.

In Section 6 we include a table of values for the sequence $\left(\omega_{m}\right)_{m=1}^{\infty}$. 


\subsection{Algorithm for generating $\beta$-expansions}

We now give details of our algorithm for generating $\beta$-expansions that we mentioned at the start of this section. In what follows we assume $\beta \in\left(1, \omega_{m}\right]$ and $x \in\left(0, \frac{1}{\beta-1}\right)$.

We define the interval $\mathcal{I}_{m}$ to be

$$
\begin{aligned}
\mathcal{I}_{m} & =\left[T_{1, \beta}^{2 m+1}\left(\frac{1}{\beta^{2}-1}\right), T_{0, \beta}^{2 m+1}\left(\frac{\beta}{\beta^{2}-1}\right)\right] \\
& =\left[\frac{-\beta^{2 m+2}+\beta+1}{\beta^{2}-1}, \frac{\beta^{2 m+2}}{\beta^{2}-1}\right] .
\end{aligned}
$$

The interval $\mathcal{I}_{m} \subset I_{\beta}$ by Property 2.5 and Property 2.6 .

Remark 2.8. The significance of the points $\frac{1}{\beta^{2}-1}$ and $\frac{\beta}{\beta^{2}-1}$ is that $T_{0, \beta}\left(\frac{1}{\beta^{2}-1}\right)=$ $\frac{\beta}{\beta^{2}-1}$ and $T_{1, \beta}\left(\frac{\beta}{\beta^{2}-1}\right)=\frac{1}{\beta^{2}-1}$. Therefore it is not possible for a point to pass over the interval $\left[\frac{1}{\beta^{2}-1}, \frac{\beta}{\beta^{2}-1}\right]$ without landing in it.

Step 1 There exists a minimal number of transformations $j(x)$ that map the point $x$ to the interval $\mathcal{I}_{m}$. This follows from the fact that $\left[\frac{1}{\beta^{2}-1}, \frac{\beta}{\beta^{2}-1}\right] \subset$ $\mathcal{I}_{m}$ and Remark 2.8, We choose a sequence of transformations $a \in$ $\Omega_{j(x)}$ such that $a(x) \in \mathcal{I}_{m}$. We fix the first $j(x)$ entries in our $\beta$ expansion of $x$ to be those uniquely determined by $a$.

Step 2 If $a(x) \in\left[\frac{1}{\beta^{2}-1}, \frac{\beta^{2 m+2}}{\beta^{2}-1}\right]$ then we can extend the $j(x)$-prefix $a$ to a $(j(x)+$ $2 m+1)$-prefix by concatenating $a$ with any element $a^{(1)} \in \Omega_{2 m+1}$ such that $\left|a^{(1)}\right|_{1} \geq m+1$. To see why $a a^{(1)}$ is a $(j(x)+2 m+1)$-prefix for $x$ we observe that

$$
\begin{aligned}
\frac{-\beta^{2 m+2}+\beta+1}{\beta^{2}-1} & =T_{1, \beta}^{2 m+1}\left(\frac{1}{\beta^{2}-1}\right) \\
& \leq a a^{(1)}(x) \\
& \leq a a^{(1)}\left(\frac{\beta^{2 m+2}}{\beta^{2}-1}\right) \\
& \leq \overbrace{T_{0, \beta}, \ldots, T_{0, \beta}}^{m} \overbrace{T_{1, \beta}, \ldots, T_{1, \beta}}^{m+1}\left(\frac{\beta^{2 m+2}}{\beta^{2}-1}\right) \\
& =T_{1, \beta}^{m+1}\left(\frac{\beta^{3 m+2}}{\beta^{2}-1}\right) \\
& =\frac{\beta^{4 m+3}-\beta^{m+2}-\beta^{m+1}+\beta+1}{\beta^{2}-1},
\end{aligned}
$$


by Lemmas 2.2 and 2.3 . The inequality

$$
\frac{\beta^{4 m+3}-\beta^{m+2}-\beta^{m+1}+\beta+1}{\beta^{2}-1} \leq \frac{\beta^{2 m+2}}{\beta^{2}-1},
$$

is equivalent to $\beta^{4 m+3}-\beta^{2 m+2}-\beta^{m+2}-\beta^{m+1}+\beta+1 \leq 0$, which is true for $\beta \in\left(1, \omega_{m}\right]$ by Property [2.4. Therefore $a a^{(1)}(x) \in \mathcal{I}_{m}$ for all $a^{(1)} \in \Omega_{2 m+1}$ such that $\left|a^{(1)}\right|_{1} \geq m+1$, which by the remarks following Proposition 2.1 implies that $a a^{(1)}$ is a $(j(x)+2 m+1)$-prefix for $x$.

If $a(x) \in\left[\frac{-\beta^{2 m+2}+\beta+1}{\beta^{2}-1}, \frac{1}{\beta^{2}-1}\right]$ then we consider elements $a^{(1)} \in \Omega_{2 m+1}$ such that $\left|a^{(1)}\right|_{0} \geq m+1$. By a similar argument it can be shown that $a a^{(1)}(x) \in \mathcal{I}_{m}$ for all $a^{(1)} \in \Omega_{2 m+1}$ such that $\left|a^{(1)}\right|_{0} \geq m+1$. As \# $\{a \in$ $\left.\Omega_{2 m+1}:\left|a^{(1)}\right|_{1} \geq m+1\right\}=\#\left\{a \in \Omega_{2 m+1}:\left|a^{(1)}\right|_{0} \geq m+1\right\}=2^{2 m}$ our algorithm generates $2^{2 m}(j(x)+2 m+1)$-prefixes for $x$.

Step 3 We proceed inductively, if $a a^{(1)}(x) \in\left[\frac{1}{\beta^{2}-1}, \frac{\beta^{2 m+2}}{\beta^{2}-1}\right]$ then we extend the $(j(x)+2 m+1)$-prefix $a a^{(1)}$ to a $(j(x)+4 m+2)$-prefix for $x$ by concatenating $a a^{(1)}$ with any element $a^{(2)} \in \Omega_{2 m+1}$ such that $\left|a^{(2)}\right|_{1} \geq$ $m+1$. Similarly, if $a a^{(1)}(x) \in\left[\frac{-\beta^{2 m+2}+\beta+1}{\beta^{2}-1}, \frac{1}{\beta^{2}-1}\right]$ then we consider elements $a^{(2)} \in \Omega_{2 m+1}$ such that $\left|a^{(2)}\right|_{0} \geq m+1$. We repeat this process indefinitely.

Remark 2.9. It is clear that by proceeding inductively our algorithm generates $2^{2 k m}(j(x)+k(2 m+1))$-prefixes for $x$, for $k \in \mathbb{N}$.

Remark 2.10. The construction of our interval $\mathcal{I}_{m}$ is somewhat arbitrary. We could have begun by choosing any interval of the form $[z, \beta z]$ for some $z \in\left(0, \frac{1}{\beta-1}\right)$. We then construct the interval $\left[T_{1, \beta}^{2 m+1}(z), T_{0, \beta}^{2 m+1}(\beta z)\right]$ to perform the role of the interval $\mathcal{I}_{m}$. By defining a similar set of polynomials to $P_{m}^{1}(x), P_{m}^{2}(x)$ and $P_{m}^{3}(x)$ and assuming our $\beta$ satisfies certain restrictions imposed by these polynomials we can ensure that $\left[T_{1, \beta}^{2 m+1}(z), T_{0, \beta}^{2 m+1}(\beta z)\right] \subset I_{\beta}$ and if $x \in\left[T_{1, \beta}^{2 m+1}(z), T_{0, \beta}^{2 m+1}(\beta z)\right]$ then $a(x) \in\left[T_{1, \beta}^{2 m+1}(z), T_{0, \beta}^{2 m+1}(\beta z)\right]$ for $2^{2 m}$ elements of $\Omega_{2 m+1}$. It would be interesting to know whether $\mathcal{I}_{m}$ is the most efficient choice of interval for this method.

We denote the set of $\beta$-expansions generated by this algorithm by $\Sigma_{\beta}(x, m)$ and the set of $k$-prefixes for $x$ generated by this algorithm by $\Sigma_{\beta}(x, m, k)$.

Property 2.11. The cardinality of $\Sigma_{\beta}(x, m, k)$ is increasing with respect to $k$. 
Property 2.12. Let $s, s^{\prime} \in \mathbb{N}$ and $s>s^{\prime}$. If $b \in \Sigma_{\beta}\left(x, m, j(x)+s^{\prime}(2 m+1)\right)$ then

$$
\begin{gathered}
\#\left\{a \in \Sigma_{\beta}(x, m, j(x)+s(2 m+1)): a_{n}=b_{n} \text { for } 1 \leq n \leq j(x)+s^{\prime}(2 m+1)\right\} \\
=2^{2 m\left(s-s^{\prime}\right)} .
\end{gathered}
$$

We now prove several technical lemmas.

Lemma 2.13. Let $\beta \in\left(1, \omega_{m}\right]$ and $x \in\left(0, \frac{1}{\beta-1}\right)$. Assume $k \geq j(x)$ then

$$
\# \Sigma_{\beta}(x, m, k) \geq 2^{2 m\left(\frac{k-j(x)}{2 m+1}-1\right)} .
$$

Proof. We have that

$$
\begin{aligned}
\# \Sigma_{\beta}(x, m, k) \geq \# \Sigma_{\beta}\left(x, m,\left((2 m+1)\left[\frac{k-j(x)}{2 m+1}\right]+j(x)\right)\right) & \geq 2^{2 m\left[\frac{k-j(x)}{2 m+1}\right]} \\
& \geq 2^{2 m\left(\frac{k-j(x)}{2 m+1}-1\right)} .
\end{aligned}
$$

Lemma 2.14. Let $l \geq j(x)$ and $b \in \Sigma_{\beta}(x, m, l)$, then for $k \geq l$

$$
\#\left\{a=\left(a_{n}\right)_{n=1}^{k} \in \Sigma_{\beta}(x, m, k): a_{n}=b_{n} \text { for } 1 \leq n \leq l\right\} \leq 2^{2 m\left(\frac{k-l}{2 m+1}+2\right)} .
$$

Proof. Consider the integers $j(x)+(2 m+1)\left[\frac{l-j(x)}{2 m+1}\right]$ and $j(x)+(2 m+1)\left(\left[\frac{k-j(x)}{2 m+1}\right]+\right.$ $1)$. We remark that $j(x)+(2 m+1)\left[\frac{l-j(x)}{2 m+1}\right]$ is an integer of the form $j(x)+s(2 m+1)$ and is less than or equal to $l$ and $j(x)+(2 m+1)\left(\left[\frac{k-j(x)}{2 m+1}\right]+1\right)$ is an integer of the form $j(x)+s(2 m+1)$ greater that or equal to $k$. It follows immediately that

$$
\begin{aligned}
& \#\left\{a=\left(a_{n}\right)_{n=1}^{k} \in \Sigma_{\beta}(x, m, k): a_{n}=b_{n} \text { for } 1 \leq n \leq l\right\} \\
& \leq \#\left\{a=\left(a_{n}\right)_{n=1}^{j(x)+(2 m+1)\left(\left[\frac{k-j(x)}{2 m+1}\right]+1\right)} \ldots\right. \\
& \quad \in \Sigma_{\beta}\left(x, m, j(x)+(2 m+1)\left(\left[\frac{k-j(x)}{2 m+1}\right]+1\right)\right): a_{n}=b_{n} \ldots \\
& \left.\quad \quad \text { for } 1 \leq n \leq j(x)+(2 m+1)\left[\frac{l-j(x)}{2 m+1}\right]\right\} \\
& \leq 2^{2 m\left(\left[\frac{k-j(x)}{2 m+1}\right]+1-\left[\frac{l-j(x)}{2 m+1}\right]\right)} \\
& \leq 2^{2 m\left(\frac{k-j(x)}{2 m+1}+1-\frac{l-j(x)}{2 m+1}+1\right)} \\
& =2^{2 m\left(\frac{k-l}{2 m+1}+2\right)},
\end{aligned}
$$

by Properties 2.11 and 2.12 . 


\subsubsection{Proof of Theorem 1.3}

We are now in a position to prove Theorem 1.3. The following proof is based upon the argument given in Example 2.7 of [3].

Proof of Theorem 1.3. By the monotonicity of Hausdorff dimension with respect to inclusion it suffices to show that $\operatorname{dim}_{H}\left(\Sigma_{\beta}(x, m)\right) \geq \frac{2 m}{2 m+1}$. It suffices to show that for any sufficiently small cover $\left\{U_{i}\right\}_{i=1}^{\infty}$ of $\Sigma_{\beta}(x, m)$ we can bound $\sum_{i=1}^{\infty} \operatorname{Diam}\left(U_{i}\right)^{\frac{2 m}{2 m+1}}$ below by a positive constant independent of our choice of cover. It is a simple exercise to show that $\Sigma_{\beta}(x, m)$ is a compact set; by this result we may restrict to finite covers of $\Sigma_{\beta}(x, m)$. Let $\left\{U_{i}\right\}_{i=1}^{N}$ be a finite cover of $\Sigma_{\beta}(x, m)$. Without loss of generality we may assume that all elements of our cover satisfy $\operatorname{Diam}\left(U_{i}\right)<2^{-j(x)}$. For each $U_{i}$ there exists $l(i)$ such that

$$
2^{-(l(i)+1)} \leq \operatorname{Diam}\left(U_{i}\right)<2^{-l(i)} .
$$

It follows that there exists $z^{(i)} \in\{0,1\}^{l(i)}$ such that $y_{n}=z_{n}^{(i)}$ for all $y \in U_{i}$, for $1 \leq n \leq l(i)$. We may assume that $z^{(i)} \in \Sigma_{\beta}(x, m, l(i))$, if we supposed otherwise then $\Sigma_{\beta}(x, m) \cap U_{i}=\emptyset$ and we can remove $U_{i}$ from our cover. We denote by $C_{i}$ the set of sequences in $\{0,1\}^{\mathbb{N}}$ whose first $l(i)$ entries agree with $z^{(i)}$, i.e.

$$
C_{i}=\left\{\left(\epsilon_{n}\right)_{n=1}^{\infty} \in\{0,1\}^{\mathbb{N}}: \epsilon_{n}=z_{n}^{(i)} \text { for } 1 \leq n \leq l(i)\right\} .
$$

Clearly $U_{i} \subset C_{i}$ and therefore the set $\left\{C_{i}\right\}_{i=1}^{N}$ is a cover of $\Sigma_{\beta}(x, m)$.

Since there are only finitely many elements in our cover there exists $J$ such that $2^{-J} \leq \operatorname{Diam}\left(U_{i}\right)$ for all $i$. We now consider the set $\Sigma_{\beta}(x, m, J)$. Since $\left\{C_{i}\right\}_{i=1}^{N}$ is a cover of $\Sigma_{\beta}(x, m)$ each $a \in \Sigma_{\beta}(x, m, J)$ satisfies $a_{n}=z_{n}^{(i)}$ for $1 \leq n \leq l(i)$, for some $i$. Therefore

$$
\# \Sigma_{\beta}(x, m, J) \leq \sum_{i=1}^{N} \#\left\{a \in \Sigma_{\beta}(x, m, J): a_{n}=z_{n}^{(i)} \text { for } 1 \leq n \leq l(i)\right\} .
$$

By counting elements of $\Sigma_{\beta}(x, m, J)$ and Lemmas 2.13 and 2.14 the following holds;

$$
\begin{aligned}
2^{2 m\left(\frac{J-j(x)}{2 m+1}-1\right)} & \leq \# \Sigma_{\beta}(x, m, J) \\
& \leq \sum_{i=1}^{N} \#\left\{a \in \Sigma_{\beta}(x, m, J): a_{n}=z_{n}^{(i)} \text { for } 1 \leq n \leq l(i)\right\}
\end{aligned}
$$




$$
\begin{aligned}
& \leq \sum_{i=1}^{N} 2^{2 m\left(\frac{J-l(i)}{2 m+1}+2\right)} \\
& =2^{\frac{2 m J+1}{2 m+1}+4 m} \sum_{i=1}^{N} 2^{\frac{-2 m(l(i)+1)}{2 m+1}} \\
& \leq 2^{\frac{2 m J+1}{2 m+1}+4 m} \sum_{i=1}^{N} \operatorname{Diam}\left(U_{i}\right)^{\frac{2 m}{2 m+1}} .
\end{aligned}
$$

Dividing through by $2^{\frac{2 m J+1}{2 m+1}+4 m}$ yields

$$
\sum_{i=1}^{N} \operatorname{Diam}\left(U_{i}\right)^{\frac{2 m}{2 m+1}} \geq 2^{\frac{-12 m^{2}-(6+2 j(x)) m-1}{2 m+1}}
$$

which is a positive constant greater than zero that does not depend on our choice of cover. Our result follows.

\section{Proof of Theorem 1.4}

Our proof of Theorem 1.4 is analogous to our proof of Theorem 1.3, as such we only give details where appropriate. Similarly we devise an algorithm for generating $\beta$-expansions, the Hausdorff dimension of the set of expansions generated by this algorithm will be greater than $\frac{1}{m+2}$ for $\beta \in\left(1, \lambda_{m}\right]$.

\subsection{The sequence $\left(\lambda_{m}\right)_{m=1}^{\infty}$}

We now give details of the sequence $\left(\lambda_{m}\right)_{m=1}^{\infty}$. Let $\lambda_{m}$ be the smallest real root of the equation

$$
x^{m+3}-x^{m+2}-x^{m+1}+1=0
$$

greater than 1 . The sequence $\left(\lambda_{m}\right)_{m=1}^{\infty}$ is well known, see [1] for details. We now make several remarks.

Remark 3.1. To see that $x^{m+3}-x^{m+2}-x^{m+1}+1$ has a real root greater than 1 , let $P_{m}(x)=x^{m+3}-x^{m+2}-x^{m+1}+1$. We observe that $P_{m}(1)=0$ and $P_{m}^{\prime}(1)<0$, this implies that $P_{m}(x)$ has a real root greater than 1 .

Property 3.2. For $\beta \in\left(1, \lambda_{m}\right]$ we have

$$
\beta^{m+3}-\beta^{m+2}-\beta^{m+1}+1 \leq 0 .
$$


Remark 3.3. Each term in $\left(\lambda_{m}\right)_{m=1}^{\infty}$ is a Pisot number, i.e. a real algebraic integer greater than 1 whose Galois conjugates are of modulus strictly less than 1. Moreover, $\lambda_{1}$ is the greatest real root of $x^{3}-x-1=0$, the first Pisot number.

Property 3.4. The sequence $\left(\lambda_{m}\right)_{m=1}^{\infty}$ is strictly increasing and it is a simple exercise to show that $\lambda_{m} \rightarrow \frac{1+\sqrt{5}}{2}$ as $m \rightarrow \infty$.

In Section 6 we include a table of values for the sequence $\left(\lambda_{m}\right)_{m=1}^{\infty}$.

\subsection{Algorithm for generating $\beta$-expansions}

We define

$$
\mathcal{I}=\left[T_{1, \beta}\left(\frac{1}{\beta^{2}-1}\right), T_{0, \beta}\left(\frac{\beta}{\beta^{2}-1}\right)\right]=\left[\frac{1+\beta-\beta^{2}}{\beta^{2}-1}, \frac{\beta^{2}}{\beta^{2}-1}\right],
$$

for $1<\beta<\frac{1+\sqrt{5}}{2}$ the interval $\mathcal{I}$ is contained within $I_{\beta}$. This interval will play a similar role to the interval $\mathcal{I}_{m}$. Before giving details of our algorithm we require the following technical Lemma.

Lemma 3.5. For $\beta \in\left(1, \lambda_{m}\right]$

$$
T_{0, \beta}^{m+1}\left(\frac{1+\beta-\beta^{2}}{\beta^{2}-1}\right) \geq \frac{1}{\beta^{2}-1} \text { and } T_{1, \beta}^{m+1}\left(\frac{\beta^{2}}{\beta^{2}-1}\right) \leq \frac{\beta}{\beta^{2}-1} .
$$

Proof. It is a simple exercise to show that

$$
T_{0, \beta}^{m+1}\left(\frac{1+\beta-\beta^{2}}{\beta^{2}-1}\right)=\frac{\beta^{m+1}+\beta^{m+2}-\beta^{m+3}}{\beta^{2}-1} .
$$

This is greater than or equal to $\frac{1}{\beta^{2}-1}$ precisely when $\beta^{m+3}-\beta^{m+2}-\beta^{m+1}+1 \leq$ 0 , this is true by property 3.2 , Our second inequality is proved similarly.

We now formalise our algorithm for generating expansions.

Step 1 Let $x \in\left(0, \frac{1}{\beta-1}\right)$, by Remark 2.8 there exists a minimal number of transformations $g(x)$ that map $x$ into the interval $\mathcal{I}$. We choose a sequence of transformations $a \in \Omega_{g(x)}$ such that $a(x) \in \mathcal{I}$. We fix the first $g(x)$ entries in our $\beta$-expansion to be those uniquely determined by $a$. 
Step 2 If $a(x) \in\left[\frac{1}{\beta^{2}-1}, \frac{\beta}{\beta^{2}-1}\right]$ then we can extend $a(x)$ to a $(g(x)+1)$-prefix by either $T_{0, \beta}$ or $T_{1, \beta}$, we then choose an element $a^{(i)}(x) \in \Omega_{m+1}$ such that $a^{(i)} \circ T_{i, \beta} \circ a(x) \in \mathcal{I}$, for $i=0,1$. This defines 2 prefixes of length $(g(x)+m+2)$ for $x$. If $a(x) \in\left[\frac{1+\beta-\beta^{2}}{\beta^{2}-1}, \frac{1}{\beta^{2}-1}\right]$ we iterate $T_{0, \beta}$ until $T_{0, \beta}^{k} \circ a(x) \in\left[\frac{1}{\beta^{2}-1}, \frac{\beta}{\beta^{2}-1}\right]$. By Lemma 3.5 and the monotonicity of the transformation $T_{0, \beta}$ we have that $k \leq m+1$. The transformation $T_{0, \beta}^{k} \circ a(x)$ defines a $(g(x)+k)$-prefix. We can extend this prefix to a $(g(x)+k+1)$-prefix by either $T_{0, \beta}$ or $T_{1, \beta}$, we then make a choice of element $a^{(i)} \in \Omega_{m+1-k}$ such that $a^{(i)} \circ T_{i, \beta} \circ T_{0, \beta}^{k} \circ a(x) \in \mathcal{I}$. This defines 2 prefixes of length $(g(x)+m+2)$ for $x$. If $a(x) \in\left[\frac{\beta}{\beta^{2}-1}, \frac{\beta^{2}}{\beta^{2}-1}\right]$ then by a similar argument to the case where $a(x) \in\left[\frac{1+\beta-\beta^{2}}{\beta^{2}-1}, \frac{1}{\beta^{2}-1}\right]$ we can formalise a method for choosing two elements of $a^{(0)}, a^{(1)} \in \Omega_{m+2}$ such that $a^{(i)} \circ a(x) \in \mathcal{I}$, for $i=0,1$. At this stage our algorithm has generated 2 prefixes of length $(g(x)+m+2)$ for $x$.

Step 3 The 2 prefixes of length $(g(x)+m+2)$ defined by Step 2 map $x$ into $\mathcal{I}$, as such we can apply Step 2 to the image of $x$ under the transformations corresponding to our 2 prefixes of length $(g(x)+m+$ $2)$. This defines 4 prefixes of length $(g(x)+2(m+2))$. We repeat this process indefinitely.

Remark 3.6. Proceeding inductively our algorithm generates $2^{k}$ prefixes of length $(g(x)+k(m+2))$ for $x$, for all $k \in \mathbb{N}$

Repeating the arguments given in Section 2 we can show that analogues of Property 2.12, Lemma 2.13 and Lemma 2.14 all hold. Theorem 1.4 then follows by an analogous argument to the one given in the proof of Theorem 1.3 .

Remark 3.7. As in the proof of Theorem 1.3 our choice of interval $\mathcal{I}$ is somewhat arbitrary. It would be interesting to know whether $\mathcal{I}$ is the most efficient choice of interval for this method.

\section{Upper bounds for the upper growth rate of $\mathcal{N}_{k}(x, \beta)$}

In this section we give bounds for the upper growth rate of $\mathcal{N}_{k}(x, \beta)$. Our main result in this section is the following theorem. 
Theorem 4.1. The supremum of the upper growth rates converges to 0 as $\beta \rightarrow 2$, i.e.,

$$
\sup _{x \in\left(0, \frac{1}{\beta-1}\right)}\left\{\limsup _{k \rightarrow \infty} \frac{\log _{2} \mathcal{N}_{k}(x, \beta)}{k}\right\} \rightarrow 0
$$

as $\beta \rightarrow 2$.

By (11) similar statements hold for $\operatorname{dim}_{H}\left(\Sigma_{\beta}(x)\right)$ and the lower growth rate of $\mathcal{N}_{k}(x, \beta)$. Theorem 4.1 can be interpreted as an analogue of Theorem 1.3 in the case where $\beta$ is close to 2 .

Proof of Theorem 4.1. Fix $m \in \mathbb{N}$. Recall that

$$
\mathcal{N}_{m}(x, \beta)=\#\left\{\left(\epsilon_{1}, \ldots, \epsilon_{m}\right) \in\{0,1\}^{m}: x-\frac{1}{\beta^{m}(\beta-1)} \leq \sum_{n=1}^{m} \frac{\epsilon_{n}}{\beta^{n}} \leq x\right\} .
$$

Let

$$
L(m, \beta)=\left\{\sum_{n=1}^{m} \frac{\epsilon_{n}}{\beta^{n}}:\left(\epsilon_{1}, \ldots, \epsilon_{m}\right) \in\{0,1\}^{m}\right\},
$$

$L(m, 2)$ is the set of dyadic rationals of degree $m$, therefore $|x-y| \geq 2^{-m}$ for all $x, y \in L(m, 2)$ such that $x \neq y$. By continuity, for each $m \in \mathbb{N}$ there exists $\delta(m)>0$, such that, for all $\beta \in(2-\delta(m), 2)$

$$
|x-y|>\frac{1}{2 \beta^{m}(\beta-1)}
$$

for all $x, y \in L(m, \beta)$ such that $x \neq y$.

It follows that any interval of length $\frac{1}{\beta^{m}(\beta-1)}$ contains at most two elements of $L(m, \beta)$. By the reformulation of $\mathcal{N}_{m}(x, \beta)$ given by (2) it follows that for $\beta \in(2-\delta(m), 2)$ any $x \in\left(0, \frac{1}{\beta-1}\right)$ has at most 2 prefixes of length $m$. Proceeding inductively we can deduce that for $\beta \in(2-\delta(m), 2)$ and $x \in\left(0, \frac{1}{\beta-1}\right)$

$$
\mathcal{N}_{k m}(x, \beta) \leq 2^{k},
$$

for all $k \in \mathbb{N}$. By a simple argument it follows that

$$
\limsup _{k \rightarrow \infty} \frac{\log _{2} \mathcal{N}_{k}(x, \beta)}{k} \leq \frac{1}{m} \text {. }
$$

As $m$ was arbitrary we can conclude our result.

The following result gives an upper bound for the upper growth rate of $\mathcal{N}_{k}(x, \beta)$ for $\beta$ close to 1 . 
Theorem 4.2. Let $m \in \mathbb{N}$ and $m \geq 2$. For $\beta \in\left(2^{\frac{1}{m}}, 2\right)$,

$$
\limsup _{k \rightarrow \infty} \frac{\log _{2} \mathcal{N}_{k}(x, \beta)}{k} \leq \frac{\log _{2}\left(2^{m}-1\right)}{m} \text { for all } x \in\left(0, \frac{1}{\beta-1}\right)
$$

Proof. It is a simple exercise to show that

$$
T_{1, \beta}^{-m}(0)=\frac{\beta^{m}-1}{\beta^{m}(\beta-1)} \text { and } T_{0, \beta}^{-m}\left(\frac{1}{\beta-1}\right)=\frac{1}{\beta^{m}(\beta-1)} .
$$

By a simple manipulation $T_{1, \beta}^{-m}(0)>T_{0, \beta}^{-m}\left(\frac{1}{\beta-1}\right)$ is equivalent to $\beta^{m}>2$. Let $\beta \in\left(2^{\frac{1}{m}}, 2\right)$ and $x \in\left(0, \frac{1}{\beta-1}\right)$, then by the above and the monotonicity of the maps $T_{0, \beta}$ and $T_{1, \beta}$ either $T_{0, \beta}^{m}(x)$ or $T_{1, \beta}^{m}(x)$ will lie outside the interval $I_{\beta}$. It follows that any $x \in\left(0, \frac{1}{\beta-1}\right)$ can have at at most $\left(2^{m}-1\right) m$-prefixes. By an inductive argument it follows that

$$
N_{k m}(x, \beta) \leq\left(2^{m}-1\right)^{k},
$$

for all $k \in \mathbb{N}$. Our result follows immediately.

\section{Application to Bernoulli convolutions}

Given $1<\beta<2$ we define the Bernoulli convolution $\mu_{\beta}$ as follows. Let $E \subset \mathbb{R}$ be a Borel set,

$$
\mu_{\beta}(E)=\mathbb{P}\left(\left\{\left(a_{1}, a_{2}, \ldots\right) \in\{0,1\}^{\mathbb{N}}: \sum_{n=1}^{\infty} \frac{a_{n}}{\beta^{n}} \in E\right\}\right),
$$

where $\mathbb{P}$ is the $(1 / 2,1 / 2)$ Bernoulli measure. For $x \in I_{\beta}$ we define the local dimension of $\mu_{\beta}$ at $x$ by

$$
d\left(\mu_{\beta}, x\right)=\lim _{r \rightarrow 0} \frac{\log \mu_{\beta}([x-r, x+r])}{\log r},
$$

when this limit exists. When the limit doesn't exists we can consider the lower and upper local dimension of $\mu_{\beta}$ at $x$, these are defined as

$$
\underline{d}\left(\mu_{\beta}, x\right)=\liminf _{r \rightarrow 0} \frac{\log \mu_{\beta}([x-r, x+r])}{\log r},
$$

and

$$
\bar{d}\left(\mu_{\beta}, x\right)=\limsup _{r \rightarrow 0} \frac{\log \mu_{\beta}([x-r, x+r])}{\log r}
$$

respectively. In [4] the authors show that the following result holds. 
Theorem 5.1. For any $\beta \in\left(1, \frac{1+\sqrt{5}}{2}\right)$ we have

$$
\bar{d}\left(\mu_{\beta}, x\right) \leq(1-\kappa(\beta)) \log _{\beta} 2,
$$

for all $x \in\left(0, \frac{1}{\beta-1}\right)$, where $\kappa(\beta)$ is as in Theorem 1.1 .

Replicating the arguments given in [4] and using the improved bounds given by Theorems 1.3 and 1.4 we can show that the following result holds.

Theorem 5.2. Let $\beta \in\left(1, \omega_{m}\right]$, then

$$
\bar{d}\left(\mu_{\beta}, x\right) \leq \frac{1}{2 m+1} \log _{\beta} 2,
$$

for every $x \in\left(0, \frac{1}{\beta-1}\right)$. Similarly for $\beta \in\left(1, \lambda_{m}\right]$, then

$$
\bar{d}\left(\mu_{\beta}, x\right) \leq \frac{m+1}{m+2} \log _{\beta} 2,
$$

for every $x \in\left(0, \frac{1}{\beta-1}\right)$.

\section{Open questions and tables for $\left(\omega_{m}\right)_{m=1}^{\infty}$ and $\left(\lambda_{m}\right)_{m=1}^{\infty}$}

Here are a few open questions:

- Does the positivity of the lower growth rate of $\mathcal{N}_{k}(x, \beta)$ imply the Hausdorff dimension of $\Sigma_{\beta}(x)$ is positive?

- Do we have equality in (1)?

- Under what conditions do we have equality in (1)?

- Is our choice of interval $\mathcal{I}_{m}$ in the proof of Theorem 1.3 the most efficient?

- Is our choice of interval $\mathcal{I}$ in the proof of Theorem 1.4 the most efficient?

The following tables list certain values of $\omega_{m}$ and $\lambda_{m}$ and their associated polynomials. 
Table 1: Table of values for the sequence $\left(\omega_{m}\right)_{m=1}^{\infty}$

\begin{tabular}{ccc}
\hline \hline$m$ & $\omega_{m}($ To 5 DP $)$ & Associated polynomials \\
\hline 1 & 1.07445 & $P_{1}^{1}(x)=x^{7}-x^{4}-x^{3}-x^{2}+x+1$ \\
& & $P_{1}^{2}(x)=x^{5}-x^{4}-x^{2}+1$ \\
& & $P_{1}^{3}(x)=x^{5}-x-1$ \\
2 & 1.02838 & $P_{2}^{1}(x)=x^{11}-x^{6}-x^{4}-x^{3}+x+1$ \\
& & $P_{2}^{2}(x)=x^{7}-x^{6}-x^{2}+1$ \\
& & $P_{k}^{2}(x)=x^{7}-x-1$ \\
3 & 1.01492 & $P_{3}^{1}(x)=x^{15}-x^{8}-x^{5}-x^{4}+x+1$ \\
& & $P_{3}^{2}(x)=x^{9}-x^{8}-x^{2}+1$ \\
& & $P_{3}^{3}(x)=x^{9}-x-1$ \\
10 & 1.00172 & $P_{10}^{1}(x)=x^{43}-x^{22}-x^{12}-x^{11}+x+1$ \\
& & $P_{10}^{2}=x^{23}-x^{22}-x^{2}+1$ \\
& & $P_{10}^{3}(x)=x^{23}-x-1$ \\
100 & 1.00003 & $P_{100}^{1}(x)=x^{403}-x^{202}-x^{102}-x^{101}+x+1$ \\
& & $P_{100}^{2}(x)=x^{203}-x^{202}-x^{2}+1$ \\
& & $P_{100}^{3}(x)=x^{203}-x-1$ \\
& &
\end{tabular}

Table 2: Table of values for the sequence $\left(\lambda_{m}\right)_{m=1}^{\infty}$

\begin{tabular}{ccc}
\hline \hline$m$ & $\lambda_{m}($ To 5DP $)$ & Associated polynomial \\
\hline 1 & 1.32472 (First Pisot number) & $x^{4}-x^{3}-x^{2}+1$ \\
2 & 1.46557 & $x^{5}-x^{4}-x^{3}+1$ \\
3 & 1.53416 & $x^{6}-x^{5}-x^{4}+1$ \\
10 & 1.61575 & $x^{13}-x^{12}-x^{11}+1$ \\
100 & 1.61804 & $x^{103}-x^{102}-x^{101}+1$ \\
\hline
\end{tabular}

Acknowledgements The author is indebted to Nikita Sidorov for much encouragement and guidance.

\section{References}

[1] J. Bertin, A. Decomps-Guilloux, M. Grandet-Hugot, M. PathiauxDelefosse and J. P. Schreiber. Pisot and Salem Numbers. Birkhuser, Basel, 1992.

[2] P. Erdős, I. Joó and V. Komornik, Characterization of the unique expansions $1=\sum_{i=1}^{\infty} q^{-n_{i}}$ and related problems, Bull. Soc. Math. Fr. 118 (1990), 377-390. 
[3] K. Falconer. Fractal Geometry: Mathematical Foundation and Applications. John Wiley, Chichester, 1990.

[4] D. J. Feng, N. Sidorov, Growth rate for beta-expansions, Monatsh. Math. 162 (2011), no. 1, 41-60.

[5] T. Kempton, Counting $\beta$-expansions and the absolute continuity of Bernoulli convolutions, Preprint.

[6] N. Sidorov, Almost every number has a continuum of $\beta$-expansions, Amer. Math. Monthly 110 (2003), 838-842.

[7] N. Sidorov, Combinatorics of linear iterated function systems with overlaps, Nonlinearity 20 (2007), 1299-1312.

Address The University of Manchester, School of Mathematics, Oxford Road, Manchester, M13 9PL. 